\title{
Numerical simulation of flow through a wellbore for horizontal wells
}

\author{
M. A. Abdulwahid ${ }^{1}$, S. F. Dakhil ${ }^{2}$ \& I. N. Niranjan Kumar $^{1}$ \\ ${ }^{1}$ Department of Marine Engineering, Andhra University, India \\ ${ }^{2}$ Department of Fuel and Energy, Basrah Technical College, Iraq
}

\begin{abstract}
An understanding of flow in long horizontal wells can be an important aspect of reservoir management. In view of this, a comprehensive study was undertaken for a pipe with a diameter of $0.022 \mathrm{~m}$ and two perforations; one on the upper surface and the other on the lower surface with $0.006 \mathrm{~m}$ diameter and $180^{\circ}$ phasing. The flow variations have been related to geometric and operating parameters. To better understand wellbore flow, Computational Fluid Dynamics CFD simulations (ANSYS CFX-13) have been used to simulate the flow at the wellbore to calculate the pressure drop, wall shear stress and turbulence kinetic energy etc. Using RNG $k-\varepsilon$ model to predict the flow behavior of turbulent pipe flow with the radial flow through perforations in the pipe wall. Comparison CFD results with the other researchers agree with them in relation to the behavior of wall shear stress, pressure drop etc.

Keywords: pipe, perforations, CFD, wellbore, turbulent flow, wall shear stress, inflow, pressure drop.
\end{abstract}

\section{Introduction}

The use of horizontal wells has become an established practice in the petroleum industry. Productivity of horizontal wells will be limited by the pressure drop within the wellbore. During the flow process from the toe-end of the horizontal well to the heel-end the drop in pressure is imminent within the wellbore. If the pressure drop within the wellbore is significant as compared to the reservoir drawdown, the reservoir drawdown along the well length will change, and consequently the production along the well length will also change. The primary 
objective is to analyze different pressure effects that contribute to overall pressure drop in horizontal wellbores beyond the wall friction effect.

The flow field in a horizontal well is different from that of the conventional pipelines due to the continuous radial inflow. In horizontal wells established with perforated liners, the reservoir fluid enters the wellbore through individual perforation holes. In addition to frictional pressure drop, pressure drop is further introduced by the continuously increasing flow velocity in the well.

The magnitude of the frictional resistance of pipe flow is determined by wall shear stress which is proportional to the velocity gradient close to the wall. Inflow through a perforation, which is perpendicular to the wellbore flow (axial flow), alters the velocity distribution and subsequently changes the wall shear stress around the perforation.

A mathematical model described for investigating how perforation distribution affects the performance of a horizontal well producing under steady state single phase flow. The model couples the Darcy flow into each perforation with one dimensional momentum equation for pipe flow. It was considered that the pressure drop in the horizontal wellbore caused by wall friction. Pressure drop due to the change in kinetic energy was neglected. Optimizing the perforation distribution was achieved using the presented method to maximize well productivity or delay gas and water coning by making the specific inflow uniform along the well [1].

Qualitative analysis has been achieved by conducting two dimensional simulations of turbulent flow in a channel with a secondary flow enters the channel through a perforation in the bottom wall of the channel. Results are compared with those from a numerical simulation of turbulent flow in a channel without perforation flow. In two dimensional simulations, since the perforation in the wall is a slot with an infinite length in the direction perpendicular to the simulated plane, only the effect of perforation flow downstream of the perforation can be analyzed. Therefore, a three dimensional simulation of turbulent flow in a pipe with inflow through a perforation has been carried out to analyze the effect of perforation flow in the circumferential direction [2].

Selecting an appropriate turbulence model has been an important issue in the numerical simulation work. The two equation $k-\varepsilon$ model has been the workhorse of engineering turbulence models for more than two decades [3] and has provided engineering accuracy in a fairly wide variety of turbulent flows [4]. However, the application of the $k-\varepsilon$ model is limited by the assumption that turbulence is isotropic. The impingement of the perforation flow with wellbore flow introduces a disturbance to both free and wall turbulent shear flow. The former takes place in the region where two streams meet and later near the wall surface. In the wall dominated region, the flow is prone to separation and thus may not be correctly simulated by the standard $k-\varepsilon$ model. 


\section{Physical and mathematical model}

\subsection{Physical model}

The physical model description is that of a pipe 3D has two perforations. The length of the pipe is $1 \mathrm{~m}$ and $0.022 \mathrm{~m}$ diameter. The perforation is $0.006 \mathrm{~m}$ diameter, the height is $0.003 \mathrm{~m}$ from the surface of the pipe with perforation phase $180^{\circ}$ to each other. The center of the perforation was placed at $0.8 \mathrm{~m}$ downstream of the pipe from the entrance. Flow through the pipe with uniform velocity is ranging from 2 to $5 \mathrm{~m} / \mathrm{s}$, and through perforation is fixed with two values of 0.121 and $0.697 \mathrm{~m} / \mathrm{s}$.

The geometry has been analyzed using 3 dimensional Computational Fluid Dynamics (CFD). Figure 1 is the unstructured computational grids, the mesh consists of 238743 nodes and 255128 hexa elements with boundary layers. Figure 1 shows the half of the pipe with one perforation because this part is symmetry with the other. The calculations were carried out with commercial finite volume code ANSYS FLUENT 13 CFX5 using a first order scheme and the turbulence with the RNG k epsilon model.

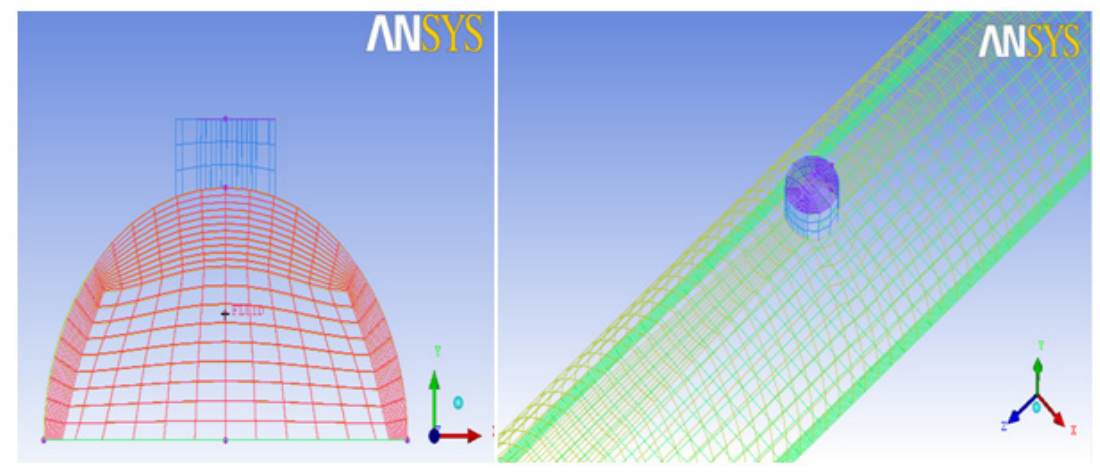

Figure 1: $\quad$ Unstructured computational grids, hexa mesh.

\subsection{Governing equations}

The Reynolds-averaged Navier-Stokes (RANS) equations govern the transport of the averaged flow quantities, with the whole range of the scales of turbulence being modeled. The RANS based modeling approach therefore greatly reduces the required computational effort and resources, and is widely adopted for practical engineering applications. An entire hierarchy of closure models is available, including Spalart-Allmaras, $k-\varepsilon$ and its variants, and $k-\omega$.

The standard, RNG and realizable $k-\varepsilon$ models have similar forms of transport equations for $k$ and $\varepsilon$. The major differences in the models include: the method of calculating turbulent viscosity, the turbulent Prandtl numbers governing the turbulent diffusion of $k$ and $\varepsilon$, the generation and destruction terms in the equation [5]. The features that are essentially common to all models 
follow, including turbulent production, accounting for the effects of compressibility. Heat and mass transfer are not considered. The steady form of mean flow is given as follows:

$$
\rho u_{j} \frac{\partial u_{i}}{\partial x_{j}}=-\frac{\partial P}{\partial x_{i}}+\frac{\partial}{\partial x_{j}}\left[\left(\mu+\mu_{t}\right) \frac{\partial u_{i}}{\partial x_{j}}\right]
$$

where $P$ pressure, $u$ X-component of velocity, $\mu$ dynamic viscosity, and $\mu_{t}$ turbulent viscosity, and $\rho$ is the density.

The RNG $k-\varepsilon$ model was derived using a statistic renormalization group theory. It is similar in form to the standard $k-\varepsilon$ model, however includes some refinements [6]

$$
\begin{aligned}
& \frac{\partial}{\partial t}(\rho k)+\frac{\partial}{\partial x_{i}}\left(\rho k u_{i}\right)=\frac{\partial}{\partial x_{j}}\left(\alpha_{k} \mu_{e f f} \frac{\partial k}{\partial x_{j}}\right) \\
& +G_{k}+G_{b}+\rho \varepsilon-Y_{M}+S_{k} \\
& \frac{\partial}{\partial t}(\rho \varepsilon)+\frac{\partial}{\partial x_{i}}\left(\rho \varepsilon u_{i}\right)=\frac{\partial}{\partial x_{j}}\left(\alpha_{\varepsilon} \mu_{e f f} \frac{\partial \varepsilon}{\partial x_{j}}\right) \\
& +C_{1 \varepsilon} \frac{\varepsilon}{k}\left(G_{k}+C_{3 \varepsilon} G_{b}\right)-C_{2 \varepsilon} \rho \frac{\varepsilon^{2}}{k}-R_{\varepsilon}+S_{\varepsilon}
\end{aligned}
$$

In these equations $G_{k}$, represents the generation of turbulence kinetic energy due to the mean velocity gradients, $G_{b}$ represents the generation of turbulence kinetic energy due to buoyancy, $Y_{M}$ represents the contribution of the fluctuating dilatation in compressible turbulence to the overall dissipation rate, $k$ turbulence kinetic energy per unit mass, $\varepsilon$ turbulent dissipation rate, $\mu_{\text {eff }}$ effective viscosity $\left(\mu+\mu_{t}\right), \quad \alpha_{k}$ and $\alpha_{\varepsilon}$ RNG $k-\varepsilon$ turbulence model constants (0.7179), and $C_{1 \varepsilon}, C_{2 \varepsilon}$ turbulence model constants, $C_{2 \varepsilon}=1.68$.

The main difference between the RNG and standard model lies in the additional term in the equation given by $R_{\varepsilon}$.

The RNG model has an additional term in its $\varepsilon$ equation that significantly improves the accuracy for rapidly varied flows. The effect of spin on turbulence is included in the RNG model, enhancing accuracy for swirling flows. The RNG theory provides an analytical formula for turbulent Prandtle numbers (Pr), while the standard $k-\varepsilon$ model uses user-specified, constant values. While the standard $k-\varepsilon$ model is a high Reynolds number model, the theory provides an analytically-derived differential method for effective viscosity that accounts for low-Reynolds-number effects. 


$$
\begin{gathered}
G_{k}=-\rho \overline{u_{i}^{\prime} u_{j}^{\prime}} \frac{\partial u_{j}}{\partial x_{i}} \\
G_{b}=\beta g_{i} \frac{\mu_{t}}{\operatorname{Pr}_{t}} \frac{\partial T}{\partial x_{i}} \\
S=\sqrt{2 S_{i j} S_{i j}} \\
\overline{S_{i j}}=\frac{1}{2}\left(\frac{\partial \overline{u_{i}}}{\partial x_{j}}+\frac{\partial \overline{u_{j}}}{\partial x_{i}}\right)
\end{gathered}
$$

where: $\overline{S_{i j}}$ the mean rate of strain tensor, $S_{k}$ source term of $k, S_{\varepsilon}$ source term of $\varepsilon$ and $\overline{u^{\prime}}$ fluctuation from the x-component of mean velocity [7].

\subsection{Assumptions and boundary condition}

The fluid chosen for simulations is water with constant density of $998.2 \mathrm{~kg} / \mathrm{m}^{3}$ and dynamic viscosity of $0.001 \mathrm{~kg} / \mathrm{m} \mathrm{s}$. The fluid is assumed as incompressible flow. The roughness of the test pipe wall is $0.03 \mathrm{~mm}$; the type of the test pipe was PVC.

A uniform velocity of water flow is introduced at the inlet pipe (axial) and inlet perforation (radial) while a pressure outlet condition is applied at the exit equal to zero. It is assumed that no-slip boundary conditions at all the walls and the effect of gravity is negligible. Water enters at a uniform temperature at $\mathrm{T}=25^{\circ} \mathrm{C}$. For the purpose of symmetry lines both velocity and pressure are kept constant.

\section{Results and discussions}

\subsection{Static pressure drop}

Static pressure drop along the center line of the pipe is shown in fig. 2. The higher value of the pressure drop appears at the entry of the pipe due to the effect entrance. When the flow was fully developed approximately $0.6 \mathrm{~m}$ downstream from the entrance, the gradient reached a constant value. A sudden pressure drop occurred at the location where the horizontal pipe flow meets the radial perforations flow. In the zone where the two streams are meeting the static pressure gradient is observed to attain a peak value and subsequently started decreasing when fluid approached the perforation and then the further decreased very steeply near the center of perforation. A further decrease in static pressure gradient is noticed in the fluid during the downstream of the perforation back 


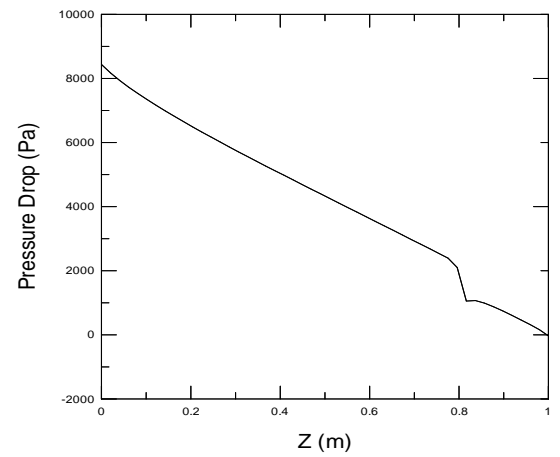

Figure 2: Static pressure distribution at the centerline pipe, with inlet1 $4 \mathrm{~m} / \mathrm{s}$ and inlet2 $0.697 \mathrm{~m} / \mathrm{s}$.

edge. The static pressure gradient increased steadily afterwards and reached a constant value close to the pipe exit.

Figure 3 depicts the comparison of pressure drop between the two cases of fixed axial flow with the varied radial flow. At inlet 1 , the velocity $4 \mathrm{~m} / \mathrm{s}$ is considered which fixed. At inlet 2 two different velocities $0.121 \mathrm{~m} / \mathrm{s}$ and $0.697 \mathrm{~m} / \mathrm{s}$ are considered. With case $10.697 \mathrm{~m} / \mathrm{s}$ it is noticed that there is a rise in static pressure gradient especially at the center of perforation and decrease in case 2 where $0.121 \mathrm{~m} / \mathrm{s}$ velocity is considered. It is attributed that for any increase in value of radial velocity at the perforation there is an increase in the pressure drop.

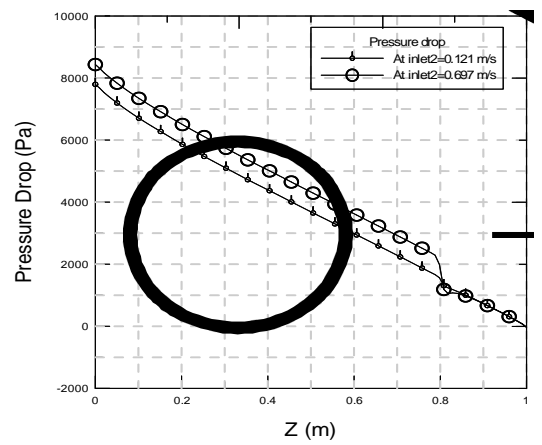

Figure 3: Static pressure distribution at the centerline pipe, with inlet1 4m/s, inlet2 $0.697 \mathrm{~m} / \mathrm{s}$ and $0.121 \mathrm{~m} / \mathrm{s}$.

Figure 4 represents the comparison of the total pressure drop in different cases with varied axial flow of $2,3,4$, and $5 \mathrm{~m} / \mathrm{s}$ and fixed radial flow of 0.697 $\mathrm{m} / \mathrm{s}$. Along the axis of the main pipe the total pressure drop (static and kinetic) is considered. In all four cases it is noticed that the total pressure drop remains same until $0.6 \mathrm{~m}$ of the pipe downstream i.e. before the perforation. It is further observed that for the increase in axial velocity value there is a corresponding increase in total pressure drop towards the exit of the pipe. 


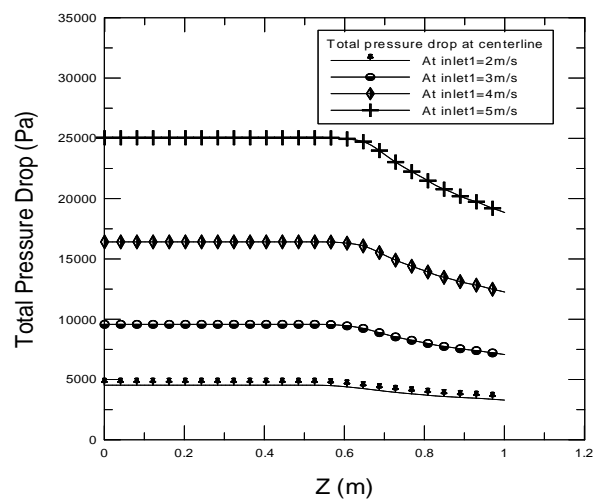

Figure 4: Comparison the total pressure drop for inlets1, 2, 3, 4, and $5 \mathrm{~m} / \mathrm{s}$ and inlet2 $0.697 \mathrm{~m} / \mathrm{s}$.

\subsection{Velocity profiles}

Velocity field around the perforations along the vertical axial plane of the main stream pipe is shown in fig. 5. It is observed that at the entrance of the pipe the velocity field is fully developed flow until the flow is reached to the perforations. The axial velocity profile at pipe exit (fig. 5(a)) indicates the flow is fully developed. The fluid flow in the radial direction flowing through perforations is colliding with the fluid flow in the axial direction is shown in fig. 5(b) [2]. The fluid velocity at the wall for the downstream from the perforation is smaller than the velocity at the wall for upstream from the entry of the pipe but absolutely no change in behavior of the velocity at the center of the entire pipe.

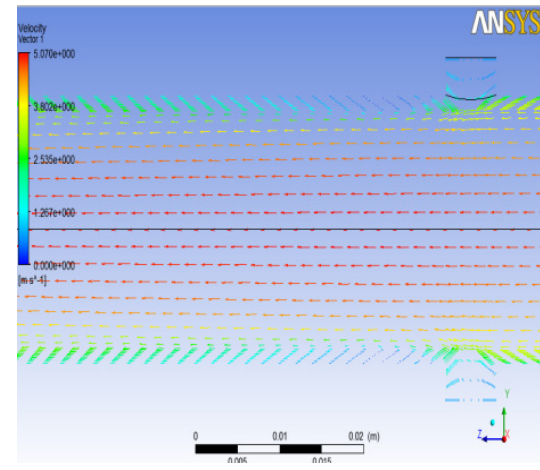

(a)

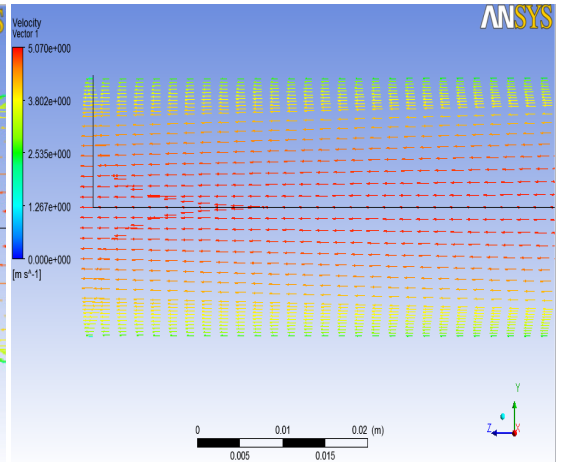

(b)

Figure 5: Velocity field around perforations for case inlet1 (4m/s) and inlet2 $(0.697 \mathrm{~m} / \mathrm{s})$. 
In order to study the velocity profiles in more detail in the main pipe, three sections are considered over the length of the pipe $Z$ where $Z=0.7 \mathrm{~m}, \mathrm{Z}=0.9 \mathrm{~m}$ and $\mathrm{Z}=1 \mathrm{~m}$ while perforations are at $\mathrm{Z}=0.8 \mathrm{~m}$. Figure 6 shows that the axial velocity before the perforation at $\mathrm{z}=0.7 \mathrm{~m}$ just before the perforation upstream and just after the perforation at $\mathrm{z}=1 \mathrm{~m}$ downstream, the flow is fully developed flow but the axial velocity at line $\mathrm{z}=0.9 \mathrm{~m}$ after the perforation is not fully developed. Turbulence flow noticed near the perforation. It is attributed to the effect of the collision of the radial flow with the axial flow.

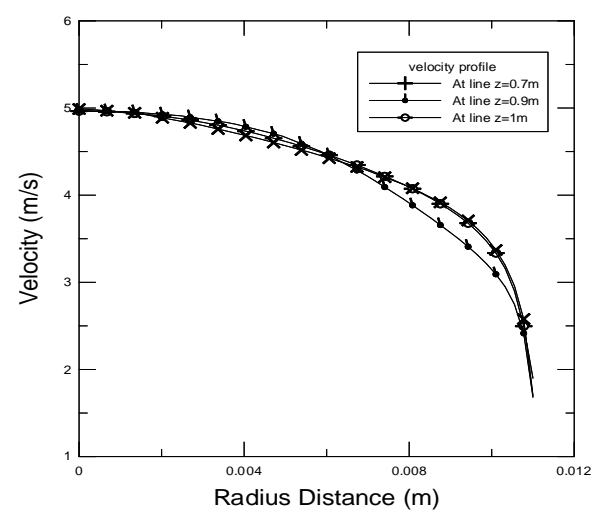

Figure 6: Velocity profile at three sections of the pipe.

\subsection{Wall shear stress distribution}

The change of the velocity gradient at the wall will alter the distribution of wall shear stress. Wall shear stress for turbulent flow is determined by both the velocity gradient at the wall and the local effective viscosity which is the sum of molecular viscosity and turbulent viscosity [8].

$$
\tau_{w}=\left(\mu+\mu_{t}\right) \frac{d u}{d y}
$$

The behavior of wall shear stress $\left(\tau_{w}\right)$ is further observed by considering two different cases one with inlet fluid of $2 \mathrm{~m} / \mathrm{s}$ in axial direction in the main pipe and with fluid velocity of $0.121 \mathrm{~m} / \mathrm{s}$ through perforation in radial direction. The second case with inlet fluid of $2 \mathrm{~m} / \mathrm{s}$ in axial direction in the main pipe and with fluid velocity of $0.697 \mathrm{~m} / \mathrm{s}$ through perforation in radial direction. Figure 7 demonstrates that in both the cases the behavior of the wall shear stress remained same and it reduced to the minimum value near the perforation. From the perforation till the downstream it started increasing but more increase in the second case where the radial stream velocity through perforation is 0.697 . 


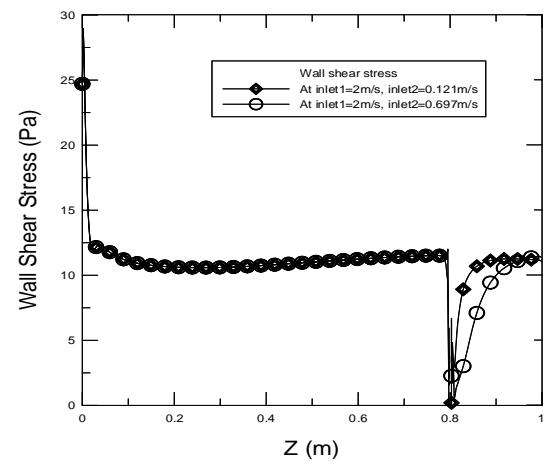

Figure 7: Wall shear stress, inlet1 2m/s, and inlet2 0.121 and $0.697 \mathrm{~m} / \mathrm{s}$.

Figure 8 demonstrated the comparison between the present work with inclusion of two perforations with pipe with the other researcher [2] who has demonstrated the behavior of wall shear stress in the entire pipe with one perforation, which appears like a hole without any perforated pipe on the upper surface and the other pipe without perforation. The wall shear stress in the present work is greater than [2] but with the similar behavior. It is concluded that the pipe with two perforations has more wall shear stress than the pipe with one perforation. Except near the perforated zone, as in the case of a pipe with perforation, the behavior of the wall shear stress in the pipe without perforation also remains the same.

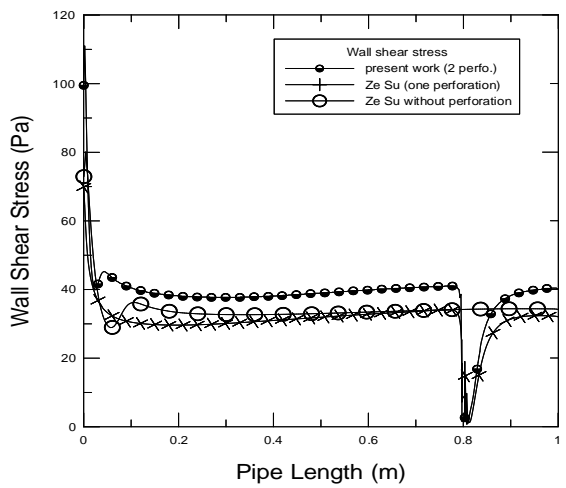

Figure 8: Comparison between the present work with [2] at $4 \mathrm{~m} / \mathrm{s}$ for inlet1 and $0.697 \mathrm{~m} / \mathrm{s}$ for inflow though perforation.

During the mass transfer process through the perforations, the time average velocity profile for turbulent flow in the pipe will be altered due to the interaction between the axial pipe flow and the perforation radial flow. Inflow lifts and expands the turbulent boundary layer and thus increases the axial velocity beyond the layer while decreases the velocity within the layer to follow 
the mass conservation law. As a consequence the axial velocity gradient near the pipe wall decreases and so does the wall friction shear stress [9].

\subsection{Turbulence kinetic energy distribution}

Turbulence kinetic energy $k$ and its dissipation rate $\varepsilon$ are two important turbulence parameters for turbulent flow. The turbulence kinetic energy represents the intensity of the velocity fluctuation of turbulent flow and is defined as [10]

$$
k=\frac{1}{2}\left(\overline{u^{\prime 2}}+\overline{v^{\prime 2}}+\overline{w^{\prime 2}}\right)
$$

The dissipation rate used in the $k-\varepsilon$ model is defined as [10]

$$
\varepsilon=v \frac{\overline{\partial u_{j}^{\prime 2}}}{\partial x_{j}^{2}}
$$

Turbulence kinetic energies are compared to demonstrate how perforation inflow affects the distribution of turbulence quantities.

The behavior of turbulence kinetic energy observed by considering two different cases one with inlet fluid of $4 \mathrm{~m} / \mathrm{s}$ in axial direction in the main pipe and with fluid velocity of $0.121 \mathrm{~m} / \mathrm{s}$ through perforation in radial direction. The second case with inlet fluid of $4 \mathrm{~m} / \mathrm{s}$ in axial direction in the main pipe and with fluid velocity of $0.697 \mathrm{~m} / \mathrm{s}$ through perforation in radial direction. Figure 9 demonstrates that in both the cases the behavior of the turbulence kinetic energy.

It is noticed that there is no difference between the two cases at the upstream side but there is a difference at downstream and shows a large difference in

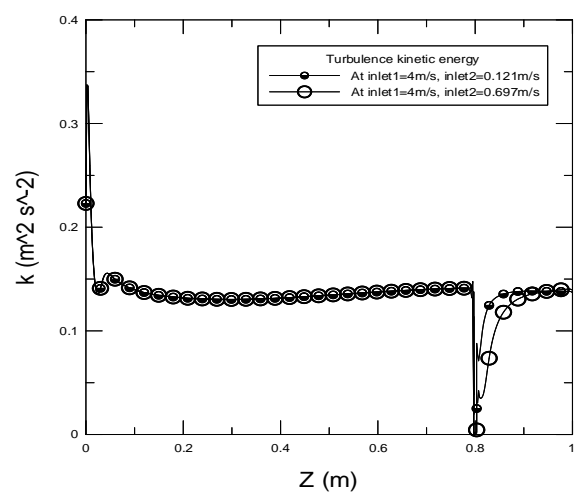

Figure 9: Turbulence kinetic energy for inlet1 $4 \mathrm{~m} / \mathrm{s}$, inlet2 0.121 and $0.697 \mathrm{~m} / \mathrm{s}$ along the entire pipe. 
turbulence intensity between the values of inflow of perforation i.e. at the lower value of radial flow through the perforation has greater value of turbulence kinetic energy but after a distance from the perforation remains equal.

\section{Conclusion}

Numerical simulations of pipe flow with two perforations, one on the upper surface and the other on the lower surface is studied. In this research work the upper half of the pipe and the perforation is only taken into account considering that the lower portion is the geometrical symmetry to the upper portion. Simulation has been carried out using the commercial CFD code ANSYS FLUENT 13-CFX and RNG $k-\varepsilon$ model was used to eliminate many limitations.

1. Noticed a very high static pressure gradient around the perforations and started decreasing the moment the fluid reached the perforation and further noticed sudden decrease near the center of the perforation. After the perforation during the downstream of the pipe, the intensity of decrease in static pressure gradient is lessened.

2. Noticed that as the velocity increases through the perforation the pressure drop increase and vice versa.

3. Study of total pressure drop (static and kinetic) is undertaken by considering four different cases with different axial velocities with fixed radial velocity. In all four cases it is noticed that the total pressure drop remains same until $0.6 \mathrm{~m}$ of the pipe downstream i.e. before the perforation. It is further observed that for the increase in axial value there is a corresponding increase in total pressure drop towards the exit of the pipe.

4. When the total pressure is considered with the varied axial flow and fixed radial flow, it remained same until just before the perforation and further with increase in axial velocity value there is a corresponding increase in total pressure drop towards the exit of the pipe.

5. At the entrance of the pipe the velocity field is fully developed flow until the perforations. When the axial and radial flows are colliding with each other, the fluid velocity at the wall for the downstream from the perforation is smaller than the velocity at the wall for upstream from the entry of the pipe but absolutely no change in behavior of the velocity at the center of the entire pipe.

6. Wall shear stress remained same and it reduced to the minimum value near the perforation when considered with fixed axial flow with varied radial flow. From the perforation till the downstream it started increasing but more increase in the case of higher radial velocities.

7. When considered with varied axial flow velocities and fixed radial flow velocity there is a reduction of wall shear stress near the perforation and 
gradually started regaining back after the perforation. The wall shear stress is completely regained back to almost original state before the fluid leaving the pipe. This reduction varied from lower velocity to higher velocity.

8. When the velocity profile is considered there is a fully developed flow notice before and after the perforation but at the perforation it is not fully developed instead a turbulent flow is noticed because of the effect of collision.

9. The increase of the number of perforations increases the wall shear stress.

10. In case of turbulent kinetic energy, the intensity started increasing at the downstream side with the decrease of fluid radial velocity at the perforation but without any change until the perforation in the upstream side.

\section{References}

[1] Landman, M. J. and Coldthorpe, W. H., "Optimization of perforation Distribution for Horizontal Wells,” SPE 23005, presented at the SPE Asia Pacific Conference, Perth, Western Australia, 1991.

[2] Ze Su, "Pressure Drop in Perforated Pipes for Horizontal Wells," Ph.D. Dissertation, Norwegian University of Science and Technology, 1996.

[3] Fluent Inc., “FLUENT User's Guide,” Version 4.3, Fluent Incorporated Lebanon, NH 03766, USA, 1995.

[4] Rodi, W., "Turbulence Models and Their Application in Hydraulics," IAHR Monograph, Third Edition, A. A. Balkema, Rotterdam, Netherlands, 1993.

[5] Qing-Shan Yang and Jian Zhang, "Simulation of Horizontally Homogeneous Atmosphere Boundary Layer Based on $k-\varepsilon$ Model Variant Models Combined With Modified Wall Functions,” The Seventh Asia Pacific Conference on Wind Engineering, November 8-12, Taipei, Taiwan, 2009.

[6] Sirnivasa Rao P., "Modeling of Turbulent Flows and Boundary Layer," Computational Fluid Dynamics, Houng Woo Oh (Ed.), In Tech, January, 2010.

[7] ANSYS Inc., ANSYS CFX-Solver Theory Guide, Version 11, ANSYS Europe, 2006.

[8] White, F. M., "Viscous Fluid Flow," Second Edition, McGraw-Hill, Inc., 1991.

[9] Liang-Biao Ouyang, Sepehr Arbabi, and Khalid Aziz, "Single Phase Fluid Flow in a Wellbore," Productivity and Injectivity of Horizontal Wells Annual Technical Report, Stanford University, 1997.

[10] Patel, V. C., W. and Scheuerer, G., "Turbulence Models for Near-Wall and low Reynolds Number Flows,” A Review,” AIAA Journal, Vol. 23, No. 9, pp. 1308-1319, September 1985. 